American Journal of Applied Sciences 2 (12): 1606-1609, 2005

ISSN 1546-9239

(c) 2005 Science Publications

\title{
Object Detection using Circular Hough Transform
}

\author{
${ }^{1}$ Mohamed Rizon, ${ }^{2}$ Haniza Yazid, ${ }^{2}$ Puteh Saad, ${ }^{2}$ Ali Yeon Md Shakaff, ${ }^{2}$ Abdul Rahman Saad, \\ ${ }^{3,4}$ Masanori Sugisaka, ${ }^{1}$ Sazali Yaacob, ${ }^{5}$ M. Rozailan Mamat and ${ }^{1}$ M. Karthigayan \\ ${ }^{1}$ School of Mechatronics Engineering, Kolej Universiti Kejuruteraan Utara Malaysia \\ Jalan Kangar-Arau 02600 Jejawi, Perlis, Malaysia \\ ${ }^{2}$ School of Computer Engineering, Kolej Universiti Kejuruteraan Utara Malaysia \\ Jalan Kangar-Arau 02600 Jejawi, Perlis, Malaysia \\ ${ }^{3}$ Advanced Research Institute for Science and Enginering, Waseda University \\ 3-4-1 Okubo, Shinjuku, Tokyo, 169-8555 Japan \\ ${ }^{4}$ Department of Electrical and Electronics Engineering, Faculty of Engineering \\ Oita University, 700 Dannoharu, Oita-shi, 870-1192 Japan \\ ${ }^{5}$ Terengganu Advanced Technical Institute (TATI), Jalan Panchor, Teluk Kalong \\ 24000 Kemaman, Terengganu, Malaysia
}

\begin{abstract}
In this study we propose a new system to detect the object from an input image. The proposed system first uses the separability filter proposed by Fukui and Yamaguchi (Trans. IEICE Japan J80-D-II. 8, 2170-2177, 1997) to obtain the best object candidates and next, the system uses the Circular Hough Transform (CHT) to detect the presence of circular shape. The main contribution of this work consists of using together two different techniques in order to take advantages from the peculiarity of each of them. As the results of the experiments, the object detection rate of the proposed system was $96 \%$ for 25 images by moving the circle template every 20 pixels to right and down.
\end{abstract}

Key words: Separability filter, circular hough transform, edge detection, mathematical morphology

\section{INTRODUCTION}

Object detection and recognition in noisy and cluttered images is challenging problem in computer vision. The goal of this research is to identify objects in an image (here we consider coconuts) by using two techniques, separability filter and the CHT. Today, there is an increase need of coconuts. Due to the necessities of the coconuts, new method had to be explored to assist the coconut gripping process. It will help to pluck the coconut from a tree using image processing techniques and it will be faster, easier and convenient than the manual plucking. There are several problems in detecting and recognizing the coconut in the image. First, the target object is obscured due to the presence of the other object which can interfere with recognition process such as the palm leaves. Second, some of the objects are overlapping between each other that make the recognition process challenging. Third, the various object positions and finally, the images itself contain noise that make the recognition process difficult without proper preprocessing and segmentation process.

The main contribution of this work consists of using together two different techniques in order to take advantages from the peculiarity of each of them: first the separability filter technique is used to obtain the best object candidates and second, the CHT is performed to detect the presence of circular shape. The separability filter was introduced by Fukui et al. ${ }^{[1]}$ and had been used in iris detection. Meanwhile the CHT is a kind of Hough transform (HT) that can extract circular objects from an image. The CHT had been used in several researches in detecting fingertips position, automatic ball recognition and iris detection for face recognition ${ }^{[2,3]}$.

Proposed system: The image with $320 \times 240$ pixels has been used through out this study. PGM (portable gray map) has been used as the input to the proposed recognition process. Figure 1 shows the block diagram of the recognition process.

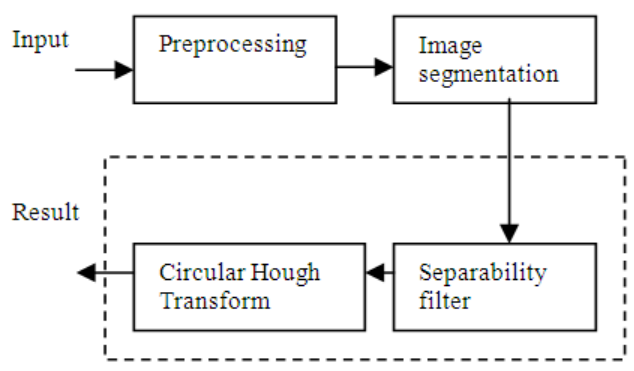

Fig. 1: The coconut recognition process

Corresponding Author: Mohamed Rizon, School of Mechatronic Engineering, Kolej Universiti Kejuruteraan Utara Malaysia, Jalan Kangar-Arau, 02600 Jejawi, Perlis, Malaysia, Tel: +60-4-9798335, Fax: +60-4-9798334 
The input image has to come across several steps before the proposed technique is performed. The image had to be enhanced using histogram equalization in preprocessing step, edge detection and mathematical morphology in segmentation step. Histogram equalization tends to increase the contrast of the image and produced a better result for region-based feature extraction. Then, the image segmentation is carried out. The edge detection and mathematical morphology had been employed to perform segmentation. The edge detection process is very important as the edge information is required for the CHT technique. Various edge detection methods have been applied for different application. Among them, Canny edge detector has been employed to the image. Canny gives thin edge compared to Sobel ${ }^{[4]}$. Beside that, the opening and closing morphology are employed to minimize the points to apply the template matching. The closing tends to close small gaps and removes much of the white pixel noise, giving a fairly clean image. Opening on the other hand tends to open small gaps or spaces between touching objects in an image. The closing has been applied then followed by the opening. The closing 'close' small gaps and make the region is clearly seen even though it consists of noise. Then, the opening took place. The opening will eliminate some of the unwanted information.

After the segmentation process, both separability filter and CHT techniques are applied. The process of separability filter and the CHT technique are described later.

The CHT is applied to image after the separability technique had been employed. When using the CHT, the radius $r$ is known in advanced because of the object's shape is peculiar feature by each other, so the radius $r$ can be treated as known parameter. In this work, the radii for the object largeness have been set to 30, 40 and 50 pixels.

In this experiment, the object of interest is the coconut throughout this research. Coconut is known in scientific as coco nucifera and is a member of Family Arecaceae (palm family). Coconut is an important plant in the lives and economies of people in South East Asia like Burma, Indonesia, Philippines and Malaysia. In Malaysia, coconut is planted either for personal use or commercialize. There are varieties of commercial coconut that had been planted in Malaysia such as Malayan Tall (MT), Malayan yellow Dwarf (MYD), Malayan Green Dwarf (MGD), Rennel Tall, Malayan Red Dwarf (MRD) and Pandan. Coconut provides almost all necessities of life like food, drink, oil, medicine, timber, thatch, mats, fuel and domestic utensils. For these, it has been called the "tree of heaven" and "tree of life". A 40 year old palm typically attains a height of $20-22 \mathrm{~m}$ and an 80 year old palm may attain a height of $35-40 \mathrm{~m}$. Due to the increasing usage of coconut, new method had to be explored to assist the coconut gripping process.

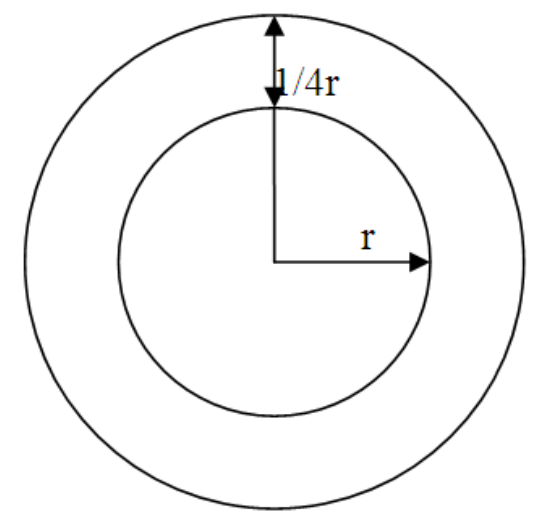

Fig. 2: The circle template to detect the object candidate

Separability filter: The separability filter is a templatebased method. The proposed technique will place the template of Fig. 2 at each point $\left(\mathrm{x}_{\mathrm{i}}, \mathrm{y}_{\mathrm{i}}\right)$ and then compute the separability between the two regions $R_{1}$ and $R_{2}$ in the template with size $r$.

Equation 3 is used to measure the separability between the two regions:

$\mathrm{A}=\sum_{\mathrm{i}=1}^{\mathrm{N}}\left(\mathrm{I}\left(\mathrm{x}_{\mathrm{i}}, \mathrm{y}_{\mathrm{i}}\right)-\overline{\mathrm{P}_{\mathrm{m}}}\right)^{2}$

$\mathrm{B}=\mathrm{n}_{1}\left(\overline{\mathrm{P}_{1}}-\overline{\mathrm{P}_{\mathrm{m}}}\right)^{2}+\mathrm{n}_{2}\left(\overline{\mathrm{P}_{2}}-\overline{\mathrm{P}_{\mathrm{m}}}\right)^{2}$

$\eta=\frac{B}{A}$

Where:

$\mathrm{N}_{\mathrm{k}}(\mathrm{k}=1,2)$ : number of pixels in $\mathrm{R}_{\mathrm{k}}$;

$\mathrm{N}=\mathrm{n}_{1}+\mathrm{n}_{2}$

$\overline{\mathrm{P}_{\mathrm{k}}}(\mathrm{k}=1,2)$ : the average intensity in $\mathrm{R}_{\mathrm{k}}$;

$\overline{\mathrm{P}_{\mathrm{m}}}$ : the average intensity in the union of $\mathrm{R}_{1}$ and $\mathrm{R}_{2}$;

$I\left(x_{i}, y_{i}\right):$ the intensity values of pixels $\left(x_{i}, y_{i}\right)$ in the union of $\mathrm{R}_{1}$ and $\mathrm{R}_{2}$.

The circles that give the local maxima of the separability $\eta$ are selected as object candidates. The points $\left(\mathrm{x}_{\mathrm{i}}, \mathrm{y}_{\mathrm{i}}\right)$ obtained after the opening morphology in the segmentation process is used as the input to the separability filter. The range of radius for the $\mathrm{R}_{2}$ region is set to $\left\{r_{L}, r_{L}+10, \ldots, r_{U}\right\}$. The template of Fig. 2 will move every 20 pixels from left to the right and 20 pixels from top to down as shown in Fig. 3.

Circular hough transform (CHT): The Hough transform (HT) and several modified versions have been recognized as robust techniques for curve detection. This method can detect object even polluted by noise. The CHT was sketched by Duda et al. ${ }^{[5]}$. 
The CHT is one of the modified versions of the HT. The CHT aims to find circular patterns within an image. The CHT is used to transform a set of feature points in the image space into a set of accumulated votes in a parameter space. Then, for each feature point, votes are accumulated in an accumulator array for all parameter combinations. The array elements that contain the highest number of votes indicate the presence of the shape. A circle pattern is described by equation 4 :

$$
\left(\mathrm{x}_{\mathrm{p}}-\mathrm{x}_{0}\right)^{2}+\left(\mathrm{y}_{\mathrm{p}}-\mathrm{y}_{0}\right)^{2}=\mathrm{r}^{2}
$$

where $x_{0}$ and $y_{0}$ are the coordinates of the center and $r$ is the radius of the circle. An example of conventional CHT is shown in Fig. 4.

The black circles indicate a set edge points within the image. Each edge point contributes a circle of radius $\mathrm{R}$ to an output accumulator space indicated by the grey circles. The output accumulator space has a peak where these contributed circles overlap at the center of the original circle. Modification to the CHT has been widely implemented to either increase the detection rate or reduce its computational complexity ${ }^{[6-8]}$. In this work, the edge orientation information is used to increase the CHT performance. This method was first

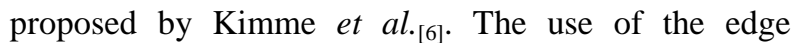
orientation information limits the possible positions of the center for each edge point. Using this method, only an arc needs to be plotted perpendicular to the edge orientation at a distance $\mathrm{R}$ from the edge point.

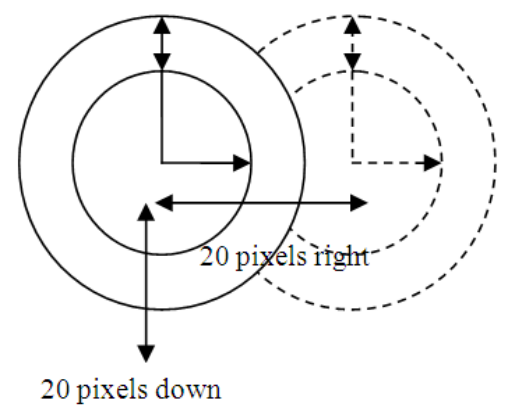

Fig. 3: Template moving

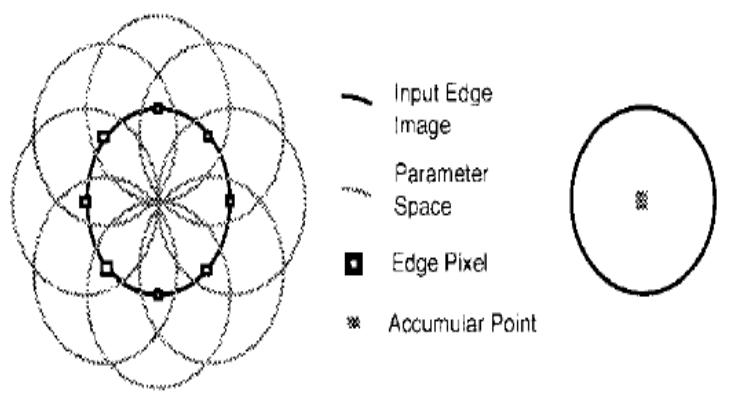

Fig. 4: The contribution of the edge points to the accumulator space

\section{EXPERIMENTAL RESULTS}

We made experiments using 25 images to evaluate the performance of the proposed system. The lower bound $r_{L}$ and the upper bound $r_{U}$ on the radius of object in the input images were set to 30 and 50, respectively. Table 1 shows the results of the experiments.

The highest success rate of the proposed system to detect the object was $96 \%$ by moving the circle template every 20 pixels to the right and 20 pixels down and the success rate was $80 \%$ even for the worst moving as shown in Table 1.

Figure 5 and 6 show examples of the images for which the proposed system gave successful results. The error occurred when the captured image was far away from the coconut palm. If the images ware taken far, the coconut image tends to be small and difficult to detect as shown in Fig. 7.

\begin{tabular}{lcc} 
Table 1: & The success rates of each template moving \\
\hline No & Template moving [pixel] & Success rate (\%) \\
\hline 1 & 10 & 88 \\
2 & 15 & 80 \\
3 & 20 & 96 \\
3 & 25 & 80 \\
4 & 30 & 80 \\
\hline
\end{tabular}

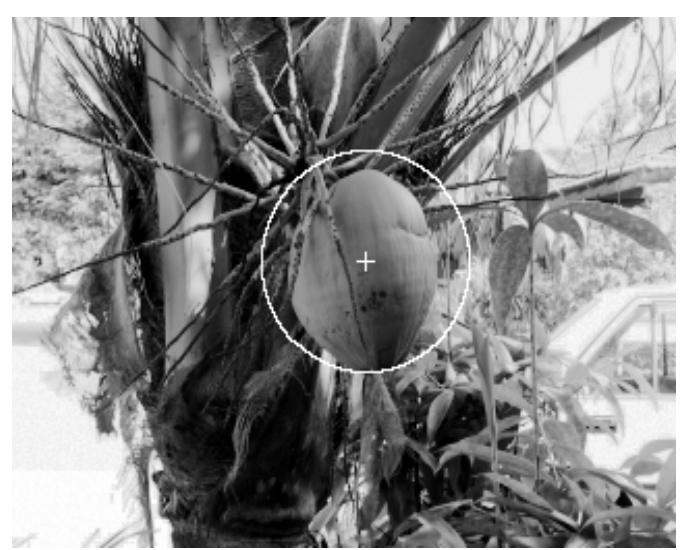

Fig. 5: Successful detection of single coconut

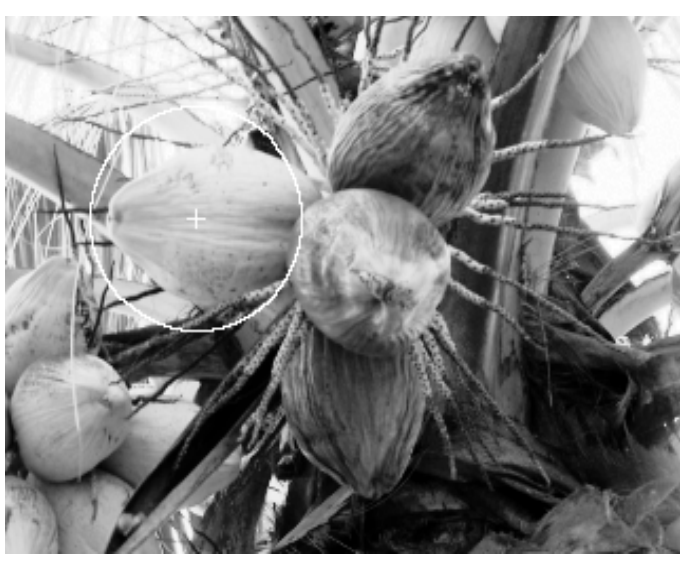

Fig. 6: Successful detection of single coconut 


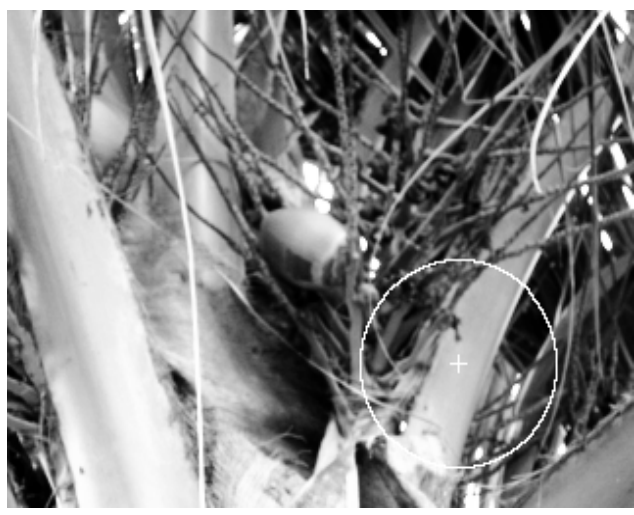

Fig. 7: Failure detection of coconut

\section{CONCLUSION}

In this research the coconut location can be detected but some constraint had been exposed. The separability filter tends to detect the possible coordinates of the coconut. Then, using the CHT, the obtained coordinates that are not considered as circle will be eliminated. The main constraint is the size of the coconut. Template is used to get the radius of the coconut largeness.

We made experiments using 25 images to evaluate the performance of the proposed system. As the results of the experiments, the highest success rate of the proposed system to detect object was $96 \%$ by moving the circle template every 20 pixels to the right and 20 pixels down and the success rate was $80 \%$ even for the worst moving.

The problem arises if the coconut image is captured far away and the image tends to be small. Beside that, some of the coconuts are in a bunch and overlaps between each other. These make the detection method difficult in carrying out the task. In this research, automatic coconut recognition can be used to assist the coconut gripping process. Future work in progress includes detection of several coconuts in a bunch and overlapping between each other.

\section{ACKNOWLEDGEMENT}

The authors wish to thank Kolej Universiti Kejuruteraan Utara Malaysia for their supports throughout this research.

\section{REFERENCES}

1. Fukui, K. and O. Yamaguchi, 1997. Facial feature points extraction method based on combination of shape extraction and pattern matching. Trans. IEICE Japan J80-D-II. 8: 2170-2177.

2. D'Orazio, T., C.Guaragnella, M. Leo and A. Distante, 2004. A new algorithm for ball recognition using circle Hough transform and neural classifier. Pattern Recognition, 37: 393-408.

3. Kawaguchi, T., D. Hidaka and M. Rizon, 2000. Detection of eyes from human faces by Hough transform and separability filter. Proc. of IEEE ICRP, 1: 49-52.

4. Yazid, H., M. Rizon, P Saad, A.Y.M. Shakaff, S. Yaacob, A.R.M. Saad and M. Sugisaka, 2005. An approach of coconuts detection using edge information. Proc. of Intl. Advanced Technology Cong., CD-ROM.

5. Duda, R.O. and P.E Hart, 1972. Use of the Hough transformation to detect lines and curves in picture. Commun. ACM, pp: 11-15.

6. Kimme, C., D. Ballard and J. Sklansky, 1975. Finding circles by an array of accumulators. Proc. ACM, 18: 120-122.

7. Ioannou, D., W. Duda and F. Laine, 1999. Circle recognition through a 2D Hough Transform and radius histogramming. Image and Vision Computing, 17: 15-26.

8. Guil, N. and E.L. Zapata, 1997. Lower order circle and ellipse hough transform. Pattern Recognition, 30: 1729-1744. 